\title{
Anesthetic management with scalp nerve block and propofol/ remifentanil infusion during awake craniotomy in an adolescent patient
} -A case report-

\author{
Bohyun Sung, Hee-Soo Kim, Jin-Woo Park, Hyo-Jin Byon, Jin-Tae Kim, and Chong Sung Kim \\ Department of Anesthesiology and Pain Medicine, College of Medicine, Seoul National University, Seoul, Korea
}

Despite of various neurophysiologic monitoring methods under general anesthesia, functional mapping at awake state during brain surgery is helpful for conservation of speech and motor function. But, awake craniotomy in children or adolescents is worrisome considering their emotional friabilities. We present our experience on anesthetic management for awake craniotomy in an adolescent patient. The patient was 16 years old male who would undergo awake craniotomy for removal of brain tumor. Scalp nerve block was done with local anesthetics and we infused propofol and remifentanil with target controlled infusion. The patient endured well and was cooperative before scalp suture, but when surgeon sutured scalp, he complained of pain and was suddenly agitated. We decided change to general anesthesia. Neurosurgeon did full neurologic examinations and there was no neurologic deficit except facial palsy of right side. Facial palsy had improved with time. (Korean J Anesthesiol 2010; 59: S179-S182)

Key Words: Adolescent, Awake craniotomy, Nerve block.

When performing a craniotomy or a lesionectomy for a brain tumor and epilepsy, if the lesion is near the motor area, the SSEP, MEP, and EMG are usually monitored [1]. However, when attempting to safely complete functional mapping with such limited electrophysiological monitoring methods, it is necessary to have the cooperation of the awake patient [2]. In the past, awake craniotomy was performed with only local anesthesia, but with the development of anesthesiology, it has been possible to perform light sedation and analgesic effects with scalp nerve blocks and intravenous anesthesia using propofol $[3,4]$.

There has been a case study made overseas of an awake craniotomy on a 9-year-old patient [5], but awake craniotomies are usually rare for patients of a young age and very troublesome. The authors of the presented case report on the anesthetic management of an awake craniotomy performed on an adolescent patient, and we aim to promote their understanding.

Received: February 11, 2010. Revised: 1st, February 17, 2010; 2nd, March 25, 2010. Accepted: April 5, 2010.

Corresponding author: Hee-Soo Kim, M.D., Department of Anesthesiology and Pain Medicine, College of Medicine, Seoul National University, 28, Yeongeon-dong, Jongno-gu, Seoul 110-744, Korea. Tel: 82-2-2072-3659, Fax: 82-2-745-5587, E-mail: dami0605@snu.ac.kr (c) This is an open-access article distributed under the terms of the Creative Commons Attribution Non-Commercial License (http:// creativecommons.org/licenses/by-nc/3.0/), which permits unrestricted non-commercial use, distribution, and reproduction in any medium, provided the original work is properly cited. 


\section{Case Report}

A 16-year-old patient of $56 \mathrm{~kg}$ and $167 \mathrm{~cm}$ underwent a craniotomy and a tumorectomy. The patient had lived a healthy life until 4 years before hospital admittance, when he was diagnosed with a glioma in the left temporal lobe from an epileptic seizure. He had been taking anti-epileptic drugs, but he started experiencing reduced drug response and had an intermittent convulsion, so surgery was decided upon. The lesion was in the left temporal lobe in the area of the sematosensory and the motor area. It was especially close to the motor area of the face and the upper extremities (Fig. 1). To allow motor mapping, we decided upon an awake craniotomy monitoring SSEP, MEP, and EMG.

In the preanesthetic visit, we evaluated the airway. There was no abnormal oral structure, and he was classified as Mallampati class 1 . We checked that neck extension was easy.

When the patient arrived on the OR reception, we explained the necessity of his awake state, anesthesia and surgery procedures, and the need for his cooperation even though it would be difficult for him. Without premedication, EKG, non-invasive blood pressure, and the pulse oximeter were monitored. A 20-gauge catheter was placed in the lt. radial artery. We then started invasive blood pressure monitoring and inserted a urinary catheter, of which he complained discomfort.

Midazolam $3 \mathrm{mg}$ and ramosetron $3 \mathrm{mg}$ were administered. The scalp nerve block was performed with $0.5 \%$ chirocaine $3 \mathrm{ml}$ as local anesthetics infused in the supraorbital, supratrochlear, auriculotemporal, lesser occipital and greater occipital nerves. A 26-gauze needle was used to compare the reduced senses with the other side of the brain. The surgeon made the head fixation

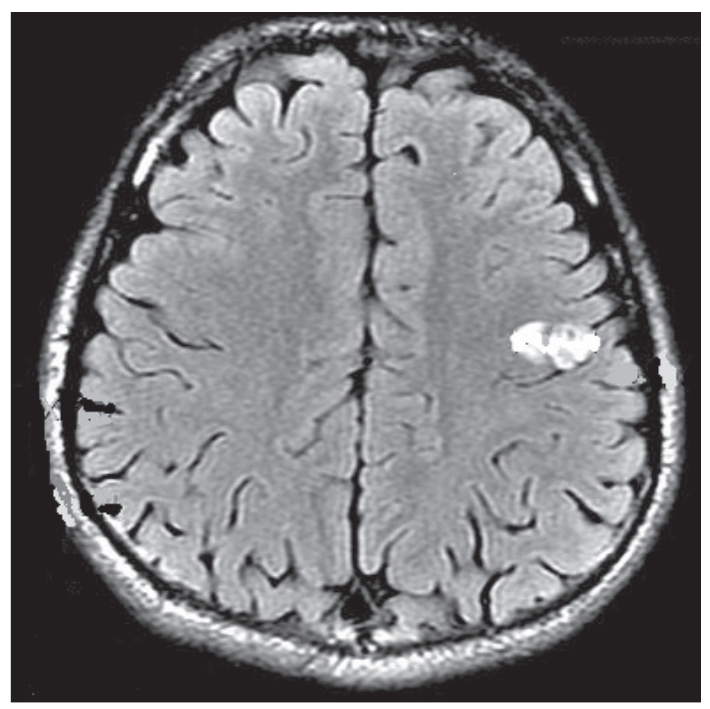

Fig. 1. MR scan shows tumorous lesion located at lt. precentral area. by first locally anesthetizing the point where the pin is to be inserted with $2 \%$ lidocaine and then using a stereotactic frame/ Mayfield head frame. When the pin was being inserted, the patient did not complain of unbearable pain. Oxygen $5 \mathrm{~L} / \mathrm{min}$ was supplied by a nasal prong. The carbon dioxide sampling line was placed in the left nostril, and the patient's breathing was monitored. The shoulders, arms, knees, and ankles were padded to reduce his discomfort during surgery. After checking that the patient's consciousness was maintained, propofol effective concentration $1 \mu \mathrm{g} / \mathrm{ml}$ and remifentanil effective concentration $1 \mathrm{ng} / \mathrm{ml}$ was maintained with target controlled infusion. A low sedative state was induced. To evaluate the patient's intraoperative consciousness level, the patient was continuously made to answer questions. The Bispectral Index was not monitored, because the area where the sensor was to be attached overlapped with the area that needed to be sterilized for surgery. During surgery, the systolic blood pressure was maintained at $110-150 \mathrm{mmHg}$, respiratory rate $8-20 / \mathrm{min}$, $\mathrm{EtCO}_{2} 33-38 \mathrm{mmHg}$, and pulse oximetry 98-100\%. During scalp cutting, dura cutting, and lesionectomy, the patient's awake state was maintained without any complications. During surgery, the patient's awake-state was maintained, and he was able to move his fingers on the command of the surgeon.

8 hours after the scalp nerve block, the brain lesion was removed. After the dural and cranial suture, the scalp was being pulled with forceps for the scalp suture, when the patient complained of intense pain, and he started to get uncontrollably agitated. With his head still fixed, the patient tried to forcefully move his body, so we were fearful of serious complications such as a cervical spine fracture. Because the tumorectomy was already completed, we judged that it was unnecessary for him to be in the awake state. With TCI, the administration of propofol was increased to the effective concentration of $4 \mu \mathrm{g} / \mathrm{ml}$, remifentanil effective concentration was increased to $4 \mathrm{ng} / \mathrm{ml}$, and rocuronium $50 \mathrm{mg}$ was administered IV. We loosened the head frame and placed the patient's head on the head rest. The incision site was covered with a sterilized $3 \mathrm{M}$ ioban. Using a laryngoscope, intubation was performed. For the rest of the surgery, general anesthesia was maintained by TIVA. Before deciding upon endotracheal intubation, using the LMA was considered, but the anesthesia screen frame was too low, that there was no room for manipulation. When we were about to wake up the patient, shaking in his right upper and lower extremities was seen. So we decided to not wake him up. Instead, midazolam $5 \mathrm{mg}$ was administered. With the patient intubated, he was ventilated using an ambu-bag and moved to the ICU. After 30 minutes in the ICU, he was extubated. 2 hours later, neurologic examination showed that his motor power in his legs were normal. A right facial palsy was observed, but his state eventually improved when checked in a follow- 
up examination. When we asked the patient what was most difficult to bear during the surgery, he answered that it was being unable to move for a long time and that he could not sleep. He could not remember experiencing unbearable pain. After the surgery, the convulsions did not reoccur. He did not experience postoperative complications, so he left 7 days later. In an outpatient follow-up, we saw that the right facial palsy improved, but his drooling tendency and nasolabial fold palsy remained.

\section{Discussion}

Awake craniotomies are opted for in order to reduce the chance of neurological defects. The anesthesiologist faces the difficulty of keeping the patient awake and cooperative and at the same time keeping the patient as comfortable as possible. The methods used for this purpose are a) the asleep-awakeasleep method and b) the monitored anesthesia care with the scalp nerve block using propofol alone or the combination of propofol and remifentanil. The asleep-awake-sleep method is convenient for the patient because it maintains the alert-state only during the times the patient must be awake. However, its drawback is that it is difficult to protect the airway and control the patient. So the monitored anesthesia care method is more commonly used. Because that propofol is an intravenous anesthetic with a low analgesic effect, using it with remifentanil, which is an opioid with a short half-life, provides more comfort to the patient. Propofol and remifentanil both have short half-lives and are easy to titrate, but their overuse or their combination use can trigger respiratory depression. Therefore, the anesthesiologist must carefully observe the patient and be alert for the occurrence of hypoventilation, apnea, or airway compromise.

Pediatric and adolescent patients are likely to be less cooperative than adults in awake craniotomy. So before the surgery, the patient's age and maturity should be considered when judging if the patient can withstand a surgery of a lengthy duration.

During awake craniotomy, the most commonly occurring accidents are seizures, agitation, nausea, vomiting, apnea, and tight brain [6]. Although there are no reports on pediatric and adolescent patients with such complications, but they may commonly occur. When seizures occur, the surgery must be stopped, and the brain must be washed with cold saline. Then, the seizures will quickly subside. Ranitidine, ondansetron, metoclopramide, and dexamethasone can be administered as premedication to prevent nausea and vomiting. In the presented case, the patient was premedicated with ramosetron, so he did not experience nausea or vomiting. When tight brain occurs, hyperventilation should be induced, and the central venous pressure must be lowered using mannitol. Pediatric and adolescent patients (as well as adult patients) may experience agitation and apnea, so the medical staff should always be ready to switch to total anesthesia. For airway patency, facial exposure is necessary, and airway securing devices such as the laryngeal mask airway and the flexible fiberoptic bronchoscope should be ready. When switching to total anesthesia, it is important to note that endotracheal intubation is not easy because the head is in the lateral rotation position, the head is fixed to the Mayfield head immobilizer, and the surgical area can become contaminated.

In the presented case, we faced great difficulty when the patient showed sudden uncontrollable agitation when the scalp was being sutured. In the case where patients suddenly move when the head is fixed with pins in an awake craniotomy, it raises the danger of skull fracturing and cervical spine fracturing. So in the cases of patients undergoing surgery where sudden agitation is expected, it is important to inform them ahead of time to reduce agitation. At the surgery stage of high pain such as cranial suturing, we regret not having relieved the patient's pain by administering nonrespiration depressant analgesics, NSAIDs. Also, if we had used the Bispectral index to evaluate his level of consciousness, the patient's airway would not have been compromised and we would have been able to induce adequate sedation $[7,8]$. Moreover, it must be noted that long surgeries and inexperienced surgeons are contraindicative to awake craniotomies [9].

Awake craniotomies are advantageous because they maintain the motor and speech function and allow for a broader scope of lesionectomies. It is expected that they will become a more popular practice in the near future. Brain tumors have a high prevalence rate in pediatric adolescent patients, so it is expected that awake craniotomies will be performed on them more in the future. There is no guarantee that pediatric-adolescent patients will be perfectly cooperative during the surgery. But safe anesthetic management can be achieved and maintained when proper explanation is made to the patient on the importance of the surgery and the importance of patient cooperation, and when intraoperative pain management and sedative measures in the form of physical and psychological support are taken.

\section{References}

1. Anastasian ZH, Ramnath B, Komotar RJ, Bruce JN, Sisti MB, Gallo EJ, et al. Evoked potential monitoring identifies possible neurological injury during positioning for craniotomy. Anesth Analg 2009; 109: 817-21.

2. Blanshard HJ, Chung F, Manninen PH, Taylor MD, Bernstein M. Awake craniotomy for removal of intracranial tumor: considerations for early discharge. Anesth Analg 2001; 92: 89-94.

3. Kim KM, Oh YS, Lee S, Kim YL, Ko H, Lee SC, et al. Anesthetic 
management for awake craniotomy with scalp nerve block and propofol/fentanil infusion. Korean J Anesthesiol 1999; 37: 57-62.

4. Costello TG, Cormack JR. Anesthesia for awake craniotomy: a modern approach. J Clin Neurosci 2004; 11: 16-9.

5. Klimek M, Verbrugge SJ, Roubos S, van der Most E, Vincent AJ, Klein J. Awake craniotomy for glioblastoma in a 9-year-old child. Anaesthesia 2004; 59: 607-9.

6. Archer DP, McKenna JM, Morin L, Ravussin P. Conscious-sedation analgesia during craniotomy for intractable epilepsy: a review of
354 consecutive cases. Can J Anaesth 1988; 35: 338-44.

7. Miner JR, Biros MH, Heegaard W, Plummer D. Bispectral electroencephalographic analysis of patients undergoing procedural sedation in the emergency department. Acad Emerg Med 2003; 10: 638-43.

8. Bell JK, Laasch HU, Wilbraham L, England RE, Morris JA, Martin DF. Bispectral index monitoring for conscious sedation in intervention: better, safer, faster. Clin Radiol 2004; 59: 1106-13.

9. Jones H, Smith M. Awake craniotomy. Contin Educ Anaesth Crit Care Pain 2004; 4: 189-92. 